\title{
May/June 2012
}

SurfNet-Technology of the Future-Today!

NSERC

CRSNG

\section{FatFonts: Combining the Symbolic and Visual Aspects of Numbers}

\section{By Miguel Nacenta, Uta Hinrichs and Sheelagh Carpendale}

FatFonts are a new type of numeric typeface designed for visualization purposes to bridge the gap between numeric and visual representations. FatFonts are based on Arabic numerals but, unlike regular numeric typefaces, the amount of ink (pixels) used for each digit is proportional to its quantitative value. This enables accurate reading of the numerical data while preserving an overall visual context.

Fatfonts are designed so that the quantity of dark pixels in a numeral character is proportional to the number it represents. For example, "2" uses twice as much ink as "1", "8" uses twice as much ink as "4", and so on.

\section{ㄱㅋㄴ드믐}

Multi-digit numbers in FatFonts are not arranged from left to right but they are nested to enable the visual representation of different ranges of data values. Each digit is scaled

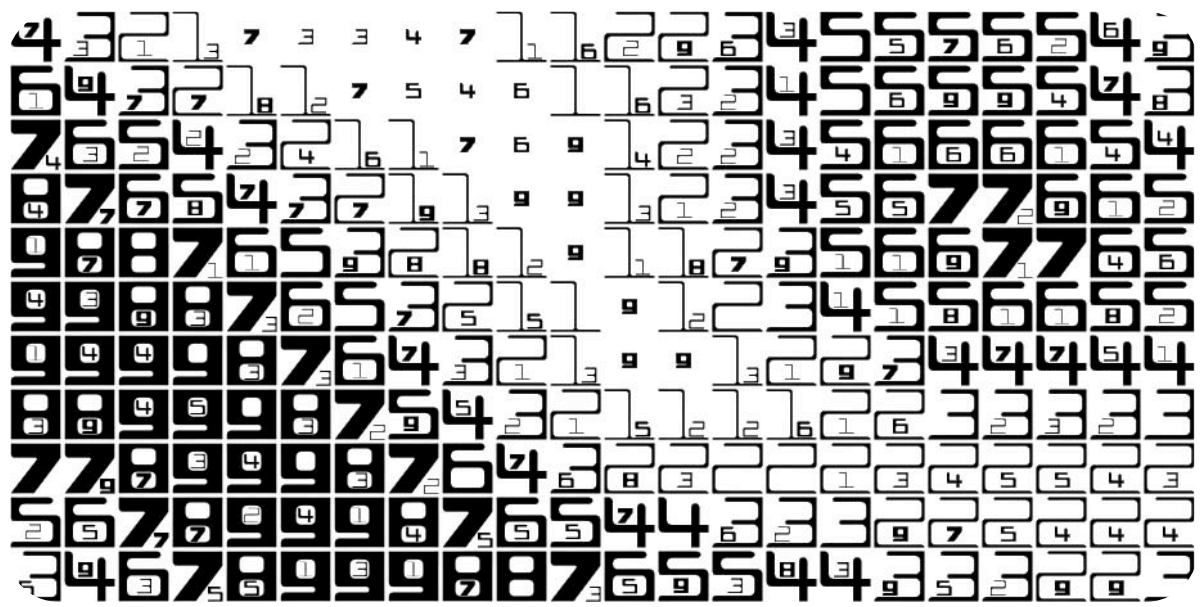

corresponding to its order of magnitude and positioned within the space of the predecessor digit. For instance, in the image above, digit 7 is nested within digit 4 , representing the number 47 .

FatFonts are specifically designed to be used on high-resolution infographic prints and large-resolution wall displays because they function interactively; up close, they represent data numerically, whereas at a distance they form a graphic image. FatFonts are useful for representing data sets where small differences in value matter, since the typeface represents them graphically.
For example, they are already being used to represent flood evacuation risks in the Netherlands and social network data.

So far, there are four different FatFont variants - these can be downloaded as .ttf or .otf files and used within regular text editors such as MS Word (see our website http:// www.fatfonts.org).

FatFonts have recently been featured in the NewScientist, UK and on Wired.co.UK

\section{In This Issue:}

- FatFonts: Combining the Symbolic and Visual Aspects of Numbers 


\section{Surface-Based Text Analysis Through Descriptive Rendering}

\section{By Daniel Chang and Christopher Collins}

The US Highway Traffic Safety Administration collects thousands of vehicle defect reports on an annual basis. When viewed collectively, these consumer-initiated complaint reports can be used to predict the quality and reliability of a specific make and model of vehicle. Using 3D graphics and intuitive interaction techniques, we propose a new approach to reviewing vehicle complaint reports with the goal of helping consumers quickly find salient facts about their vehicles.

Our visualization software supports tasks such as comparing the reliability of different makes and models of vehicles or examining the root causes of vehicle defects over time. Standard tools for analyzing this data simply return hundreds of complaint reports in a lengthy list. We encode these data as graphic effects onto a 3D illustration of a car, putting visual emphasis on areas of the most interest in the complaint reports. More frequently mentioned

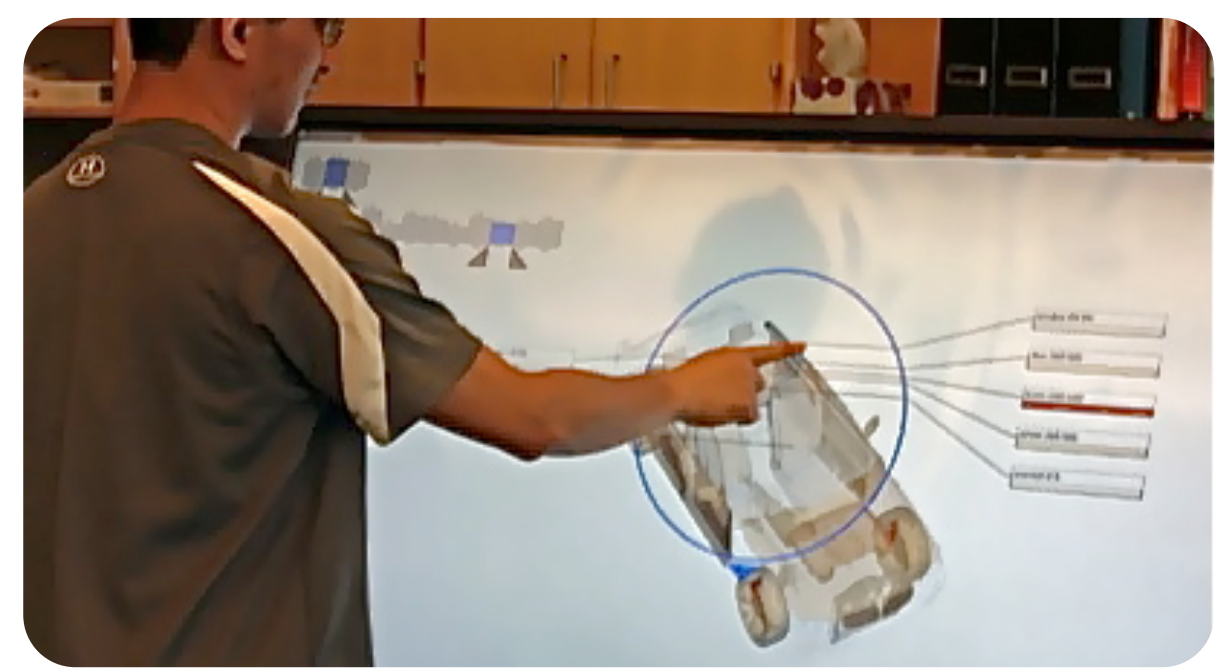

parts visually "pop out" from the rest of the model, drawing attention to problem areas quickly. Users can change the view and filter the data using interactive widgets to reveal trends such as an increase in component failures over a period of time.

In addition to the example of car buyers using the software to research vehicle defects prior to making a purchase, we expect this software to be of use to automotive engineers and quality assurance personnel. To support an ambient visualization scenario we are implementing multitouch interactivity for working with the visualization on a large display screen, with the support of a SurfNet special project grant. This project addresses the challenge of integrating multi-touch surface interactivity for 3D scientific visualization and $2 \mathrm{D}$ information visualization into a single interface.

\section{In the News:}

\section{CKWS Television (Kingston) recently}

For SurfNet contact information please go to: www.nsercsurfnet.ca/pmwiki.php? $n=$ SurfNet. Contact

visited the EQUIS lab to report on the

Liberi Live project. Liberi Live is a platform that allows players to both design and play games in realtime. Check out the video where Nick Graham, Irina Schumann (an exchange student from the LEIF project), and Zi Ye demonstrate Liberi Live.

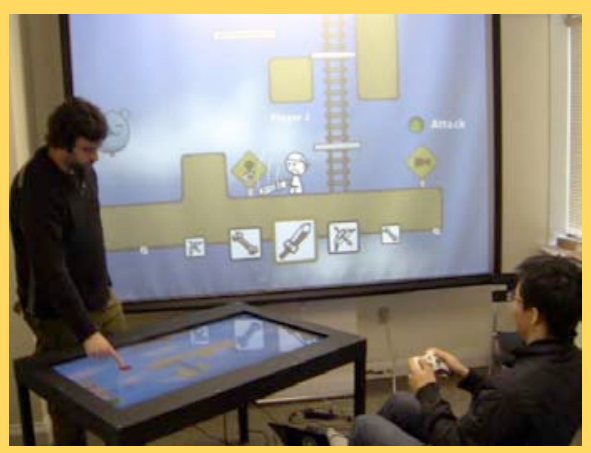

Liberi Live Demonstration

http://www.youtube.com/watch?v=r4KhxvO+SQI\&feature=player_embedded 